\title{
Study on symmetry of second type curve integral
}

\author{
Ming Wan \\ Nanchang Institute of Science \&Technology, Nanchang 330108
}

Keywords: second type curve integral, Stokes formula, set up differential, integral

\begin{abstract}
: the second type curve integral is defined function in the plane or space curve segmentpoints. This paper mainly use Stokes formula. Full explanation of the second type curve integraltechnique. Combined with concrete examples andsummarize its references the characteristics, application of familiar with various methods.
\end{abstract}

\section{Introduction}

The curve integral of type second has obvious geometric meaning and physical meaning compared with the first type curve integral,The first type curve integral can be regarded as the calculation of definite integral, The calculation of the second type curve integral is also called the integral of the coordinate, which has the directivity of the first type curve integral, the calculation is complex, and the physical meaning is obvious, This has important applications in physics. For different types of the integrand, the corresponding calculation methods are also different. In order to make the calculation more simple, this paper expounds the calculation method of line integral of the second type, not only through the parameter equations into integral calculation,But the plane curve can also through the Green formula into the calculation of double integral of the second kind of curvilinear integral can also according to the symmetry of parity two kinds of simplified calculation or by using mathematical software Mathmatic to calculate.

\section{Literature review The evaluation of domestic and foreign research status.}

From the above related can be seen, the researchers calculate many of the second type curve integral is discussed from different aspects, but only from one aspect of discussion, most of the literature are not combined with mathematical software Mathmatic space drawing and calculation. Second curve integral has a variety of ways, then it is the specific method of calculation? In this paper, on the basis of the literature on this issue are summarized, the mathematical software Mathmatic is also used in them, and giving some targeted and typical examples.On the plane, the bivariate $\mathrm{F}(\mathrm{X}, \mathrm{Y})$ function of smooth curve $\mathrm{C}(\mathrm{A}, \mathrm{B})$ on the plane divides the curve into a rational one, which is divided into two parts: $\widehat{A_{0}} A_{1}, \widehat{A_{1}} A_{2}, \ldots, A_{n-1} A_{n}$, inside, $A_{0}=A, A_{n}=B$.

Shad small arc K, $A_{k-1} A_{k}$ and $\overrightarrow{A_{k-1} A_{k}}$ The long distance between $\mathrm{X}$ and $\mathrm{Y}$ the axis and axis is identical with $\Delta x_{k}$ and $\Delta y_{k}$.At small arc K, $A_{k-1}{ }_{1} A_{k}$ Take a bit $\overrightarrow{E_{k}}\left(\varepsilon_{k}, \eta_{k}\right)$ in sum. 


\section{Computation of the line integrals of the second form}

Five calculation methods of curve integral are introduced: direct calculation, green formula, curve and path independent calculation, using symmetry and symmetry and mathematical software mathmatic.

Threshold: the starting point of the xian—, the upper limit —zhongdian. we illustrate the application of this method through several examples.Example $\int_{L}^{\int} x y d x$, It is a L parabola. $y^{2}=x$ since $^{A(\mathbf{1},-\mathbf{1})}$ to $B(\mathbf{1}, \mathbf{1})$.

Jieruoqu as x parameters, then: $\overparen{A}+O B$,

$$
\begin{aligned}
& \text { AO }: y=-\sqrt{x}, x: \mathbf{1} \rightarrow \mathbf{0}, \\
& \text { OB }: y=\sqrt{x}, x: \mathbf{0} \rightarrow \mathbf{1}, \\
& \therefore \quad \int_{L} x y d x=\int_{1}^{0} x y d x+\int_{0}^{1} x y d x \\
& =\int_{1}^{0} x(-\sqrt{x}) \mathrm{d} x+\int_{0}^{1} x \sqrt{x} \mathrm{~d} x \\
& =2 \int_{0}^{1} x^{3 / 2} \mathrm{~d} x=\frac{4}{5},
\end{aligned}
$$

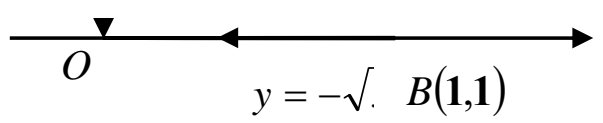

If taken y as a parameter,

$$
\begin{aligned}
& \text { so } \int_{L} x y \mathrm{~d} x=\int_{-1}^{1} y^{2} y\left(y^{2}\right)^{\prime} \mathrm{d} y, \\
& =\mathbf{2} \int_{-1}^{1} y^{4} \mathrm{~d} y=\frac{\mathbf{4}}{\mathbf{5}}
\end{aligned}
$$

\section{Using the mathematical software mathmatic to calculate}

The approximate numerical calculation of definite integral in Mathematica system is nintegrate, its calling format is as followsNIntegrate $[f(x),\{x, a, b\}]$,

In OFF-LINE-SINEWAVE is the integral function, for the integral variable, the lower limit, for the integral upper limit, sometimes can be obtained, we use Mathematica to calculate the example 1, example 2, example 4, and the Li 5. Li solution if selected as parameter input.I1= NIntegrate $\lfloor-\sqrt{x} * x,\{x, \mathbf{1}, \mathbf{0}\}\rfloor$,

I1 = NIntegrate $\left\lfloor x^{*} \sqrt{x},\{x, \mathbf{0}, \mathbf{1}\}\right\rfloor$, $\mathrm{I}=\mathrm{I} 1+\mathrm{I} 2$. 
Get the 0-8. ruoxuanqu as parameter inputIntegrate[y^2*y*2y, $\{\mathrm{y},-1,1\}]$ result $\frac{\mathbf{4}}{\mathbf{5}}$. Ifwe enter NIntegrate[$\left[\mathrm{y}^{\wedge} 2^{*} \mathrm{y}^{*} 2 \mathrm{y},\{\mathrm{y},-1,1\}\right]$.

$$
\text { result } \mathbf{0} \cdot \mathbf{8} \text {. }
$$

\section{Conclusion}

Main points of the second type curve integral integrand function has a variety of types, students in the time to do the students vulnerable to the limitations of thinking, for different types of somehow, in addition, for some types of topics to a given problem, although one can solve, but sometimes seem tedious, then it can think of other methods, so that the problem becomes simple. We can also use the mathematical software Mathematica to solve the simple calculation.

The implications for different types of the integrand, the calculation method of the second type curve integral made a systematic analysis on the easy to use what method calculation and flexible use of computational techniques are helpful in the title, so as to further improve the students' ability of problem solution, formula and theorem memory good.

\section{Reference}

[1] Liu Yulian. Mathematical analysis[M]. Beijing: Higher Education Press, 2003:11-19.

[2] Fullview dragon. Math selection and generaltechniques [M]. Beijing: Higher Education Press, 2000:89-99.

[3] Xue Jiaqing. Higher mathematicsexam Jingbian (Science) [M]. Shenyang: Northeastern University press, 2000:198-200.

[4] Liu Guojun, Chen Shaoye. Mathematical analysistutorial [M]. Beijing: Higher Education press, editedby 2002:111-119.

[5] Ding Xiaoqing.21 centurycollege teaching materials (EngineeringMathematics Analysis) [M]. Beijing: Science Press, 2008:61-75.

[6] Liu Lianfen. Teaching exploration ofcurvilinear integral and surface integral [J]. Journalof Chongqing Jiaotong College, 1988, (7): 3-4.

[7] Yu Xiaohua. A class can be directly intomathematics, repeated integral integral surface [J]. University 1999 (5): King 2-6.

[8] Jingke. Methodsand techniques of Higher Mathematics in Beijing[M]. Chinese: Forestry Publishing House, 2002:111-119.

[9] Youzeng. Hu line integral andsurface decomposition technique [J]. problem ofnonferrous metals in higher education research, 1995, (4): 4-6. 